\title{
Four new earthworm species of the genus Amynthas Kinberg (Oligochaeta: Megascolecidae) from the island of Hainan and Guangdong Province, China
}

\author{
Jibao Jiang ${ }^{\mathrm{a}, \mathrm{b}}$, Jing Sun ${ }^{\mathrm{a}, \mathrm{b}}$, Qi Zhao ${ }^{\mathrm{a}, \mathrm{b}}$ and Jiangping Qiu ${ }^{\mathrm{a}, \mathrm{b} *}$ \\ ${ }^{a}$ School of Agriculture and Biology, Shanghai Jiao Tong University, Shanghai, China; \\ ${ }^{b}$ Key Laboratory of Urban Agriculture (South), Ministry of Agriculture, Shanghai, China
}

(Received 27 November 2012; accepted 2 June 2014; first published online 11 July 2014)

\begin{abstract}
This paper describes four new species of morrisi-group earthworms from Hainan and Guangdong Provinces, China: Amynthas instabilis sp. nov, Amynthas dilatatus sp. nov, Amynthas infuscuatus sp. nov. and Amynthas qiongzhongensis sp. nov. All four species have two pairs of spermathecal pores in 5/6-6/7; male pores in XVIII, 0.33 circumference ventrally apart, each on the top of a slightly raised porophore, surrounded by several tiny genital papillae, in a pulvinate pad with three to six circular folds. Characters of the spermathecae, prostate glands and other anatomical features easily distinguish the new species from earthworms previously reported from the morrisi-group.

http://zoobank.org/urn:1sid:zoobank.org:pub:545E9152-C826-44A2-949E-402434 D8493D.
\end{abstract}

Keywords: morrisi-group; new species; genetic distances; cytochrome oxidase subunit I gene; barcode

\section{Introduction}

The morrisi-group is defined as Amynthas with intersegmental spermathecal pores, first spermathecal pores at 5/6, two thecal segments and holandry. Sims and Easton (1972) included 30 species in this group and, since 1972, nine more species from the morrisigroup have been reported from China: Amynthas nanulus (Chen et Yang 1975) (Chen et al. 1975), and Amynthas parvus (Chen and Hsu 1977) from mainland China, Amynthas diaoluomontis Qiu and Sun, 2009, Amynthas octopapillatus Qiu and Sun, 2009, Amynthas zhangi Qiu and Sun, 2009, Amynthas lingshuiensis Qiu and Sun, 2009, Amynthas endophilus Zhao and Qiu, 2013, Amynthas fluviatilis Zhao and Sun, 2013, Amynthas fucatus Zhao and Jiang, 2013 from Hainan Island of China (Sun et al. 2009; Zhao et al. 2013). Amynthas piagolensis Hong and James, 2001, Amynthas taebaekensis Hong and James, 2001, Amynthas naejangensis Hong and James, 2001, Amynthas draconis Hong and James, 2001, Amynthas assimilis Hong and Kim, 2002, Amynthas angulatus Hong, 2007 and Amynthas dabudongensis Hong and James, 2009 have been reported from Korea (Hong and James 2001, 2009; Hong and Kim 2002; Hong 2007).

Earthworms of the morrisi-group from the island of Hainan were first reported by Chen Yi (Chen 1938), and included Amynthas morrisi (Beddard 1892), Amynthas puerilis (Chen 1938), Amynthas hainanicus (Chen 1938), Amynthas oculatus (Chen

\footnotetext{
*Corresponding author. Email: jpq@sjtu.edu.cn
} 
1938), Amynthas monoserialis (Chen 1938) and Amynthas sinuosus (Chen 1938). Since then, Sun et al. (2009) have reported four new species while Zhao et al. (2013) reported three new species. In total, 17 species of the morrisi-group have been recorded from Hainan. There are no earlier reports of morrisi-group earthworms from Guangdong Province, which is located just north of Hainan Province and separated from it by Qiongzhou Strait. During the years 2006, 2010 and 2011, we made a broad earthworm collection during field surveys in southern China, and discovered more new species of the morrisi-group.

In this paper, we describe four new species from Xiangtoushan National Nature Reserve of Guandong, Jianfengling National Nature Reserve and Diaoluoshan National Nature Reserve of Hainan, including Amynthas instabilis sp. nov., Amynthas dilatatus sp. nov., Amynthas infuscuatus sp. nov. and Amynthas qiongzhongensis sp. nov. Amynthas instabilis sp. nov. was the only species found in both Hainan and Guangdong Provinces.

\section{Material and methods}

The earthworms collected in 2006 were anaesthetized in 10\% ethanol solution, and preserved in 10\% formalin solution. The earthworms collected in 2010-11 were anaesthetized in 10\% ethanol solution, and preserved in $95 \%$ ethanol solution. Holotypes and paratypes are deposited in the Shanghai Natural History Museum.

The earthworms collected in 2010-11 and preserved in 95\% ethanol solution, are suitable for molecular analysis. DNA was extracted from several specimens of A. instabilis, A. qiongzhongensis and A. morrisi (one clitellate, SC201006-03) using the E.Z.N.A. Mollusc DNA Kit (Omega Bio-tek, Norcross, GA, USA). The gene cytochrome oxidase subunit I, (COI), was amplified. Primers used in the research were 5'-GGTCAACAAATCATAAAGATATTGG-3' and 5'-TAAACTTCAGGG TGACCAAAAAATCA-3' (Folmer et al. 1994), or 5'- GGTCAACAAATCAT AAAGATATTGG-3' and 5'-TATACTTCTGGGTGTCCGAAGAATCA-3' (Bely and Wray 2004). Sequencing was performed in the Beijing Genomics Institute (Shanghai, China).

Sequences were aligned with Clustal $\mathrm{X}$, and then pairwise distances between these species were calculated using the Kimura two-parameter model of DNA evolution with MEGA 5.

\section{Results}

The COI mitochrondrial DNA gene, considered as a barcode for earthworm identification (Huang et al. 2007; Novo et al. 2010), is an effective complement to morphological analyses.

Comparisons of COI gene sequences among $A$. instabilis sp. nov., A. qiongzhongensis sp. nov. and $A$. carnosus, $A$. robustus and $A$. triastriatus (Table 1), which belong to other species-groups and have similar male pores and genital markings and different numbers of spermathecal pores, indicated that the new species greatly differ from these last species. Pairwise distances of COI (Table 2) for A. instabilis, A. qiongzhongensis and other species show very low intraspecific values $(0-0.16 \%)$, whereas distances between $A$. instabilis and $A$. qiongzhongensis were $13.59-13.79 \%$. Among $A$. instabilis and the other three species they were $18.74-21.74 \%$. Among A. qiongzhongensis and the other three species they were $17.05-19.72 \%$. According to Chang and James (2011), values above 10-15\% 
Table 1. Species with molecular data used in this study.

\begin{tabular}{|c|c|c|c|c|}
\hline Species & Species no. & Locality & Reference & $\begin{array}{l}\text { Accession } \\
\text { no. of COI } \\
\text { in NCBI }\end{array}$ \\
\hline Amynthas instabilis 1 & C-HN201001-01 & China: Hainan & This paper & JX126729 \\
\hline Amynthas instabilis 2 & C-HN201101-03 & China: Hainan & This paper & JX126727 \\
\hline Amynthas instabilis 3 & C-GD201105-03 & $\begin{array}{l}\text { China: } \\
\text { Guangdong }\end{array}$ & This paper & JX126730 \\
\hline $\begin{array}{l}\text { Amynthas } \\
\quad \text { qiongzhongensis } 1\end{array}$ & C-HN201008-01 & China: Hainan & This paper & JX126731 \\
\hline $\begin{array}{l}\text { A mynthas } \\
\quad \text { qiongzhongensis } 2\end{array}$ & C-HN201114-02 & China: Hainan & This paper & JX126728 \\
\hline Amynthascarnosus & & Japan: Osaka & $\begin{array}{c}\text { (Minamiya 2010, } \\
\text { unpublished) }\end{array}$ & AB542454 \\
\hline Amynthas morrisi & C-SC201006-03 & China: Sichuan & This paper & KF021247 \\
\hline Amynthas robustus & & China & (Huang et al. 2007) & EF077571 \\
\hline $\begin{array}{l}\text { Amynthas } \\
\text { triastriatus }\end{array}$ & & China & (Huang et al. 2007) & EF077607 \\
\hline
\end{tabular}

most probably indicate different species. Although the evaluation criterion is uncertain, it is clear that the new species and other species in Table 2 have large genetic divergences. Also, there is little difference between individuals of the same species.

\section{Systematics}

Amynthas instabilis Qiu and Jiang sp. nov.

(Figure 1)

\section{Material}

Holotype. 1 clitellate (C-HN011-02A): China, Hainan Island, Jianfengling National Nature Reserve (18 $\left.43^{\prime} 32^{\prime \prime} \mathrm{N}, 108^{\circ} 53^{\prime} 33^{\prime \prime} \mathrm{E}\right), 860 \mathrm{~m}$ elevation, black sandy soil under shrubbery beside road, 4 July 2006, J.X. Li and W.X. Zhang coll.

Paratypes. 60 clitellates (C-HN011-02B) and 3 clitellates (C-HN011-03)with the same data as for holotype; 1 clitellate (C-HN014-03): China, Hainan Island, Jianfengling National Nature Reserve (1843'58" N, 108 53'10" E), 890 m elevation, sandy soil under evergreen forest, 4 July 2006, J.P. Qiu and M.B. Bouché colls; 8 clitellates (C-HN015-01): China, Hainan Island, Jianfengling National Nature Reserve (1843'56" N, 108 53'13" E), 895 m elevation, cinnamon soil under tropical rainforest vegetation, 4 July 2006, J.P. Qiu, M.B. Bouché and X.L. Zhang colls. 2 clitellates (C-HN201001-01): China, Hainan Island, Diaoluoshan National Nature Reserve $\left(18^{\circ} 42^{\prime} 32^{\prime \prime} \mathrm{N}, 109^{\circ} 50^{\prime} 25^{\prime \prime} \mathrm{E}\right), 631 \mathrm{~m}$ elevation, black sandy soil under arbor vegetation, 23 July 2010, J.B. Jiang and Y.Z. Guo coll. 1 clitellate (C-HN201101-03): China, Hainan Island, Diaoluoshan National Nature Reserve $\left(18^{\circ} 44^{\prime} 18^{\prime \prime} \mathrm{N}, 108^{\circ} 52^{\prime} 01^{\prime \prime} \mathrm{E}\right), 840 \mathrm{~m}$ elevation, yellow soil under broadleaved evergreen forest, 23 May 2011, J.P. Qiu, J.B. Jiang, Q. Zhao, D. Cluzeau and W.K. 
4 J. Jiang et al.

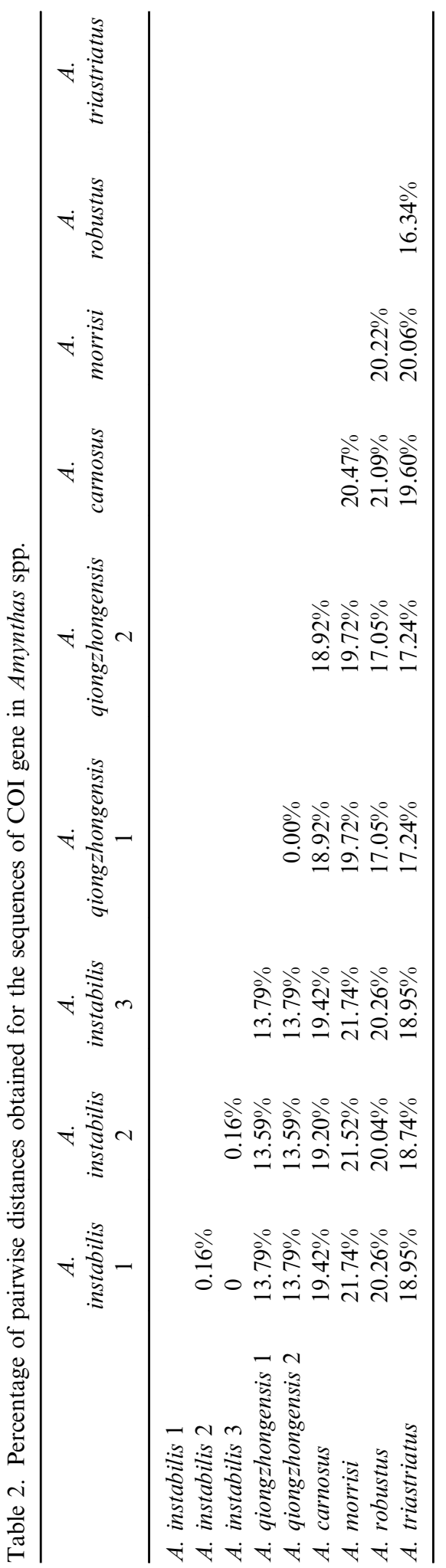



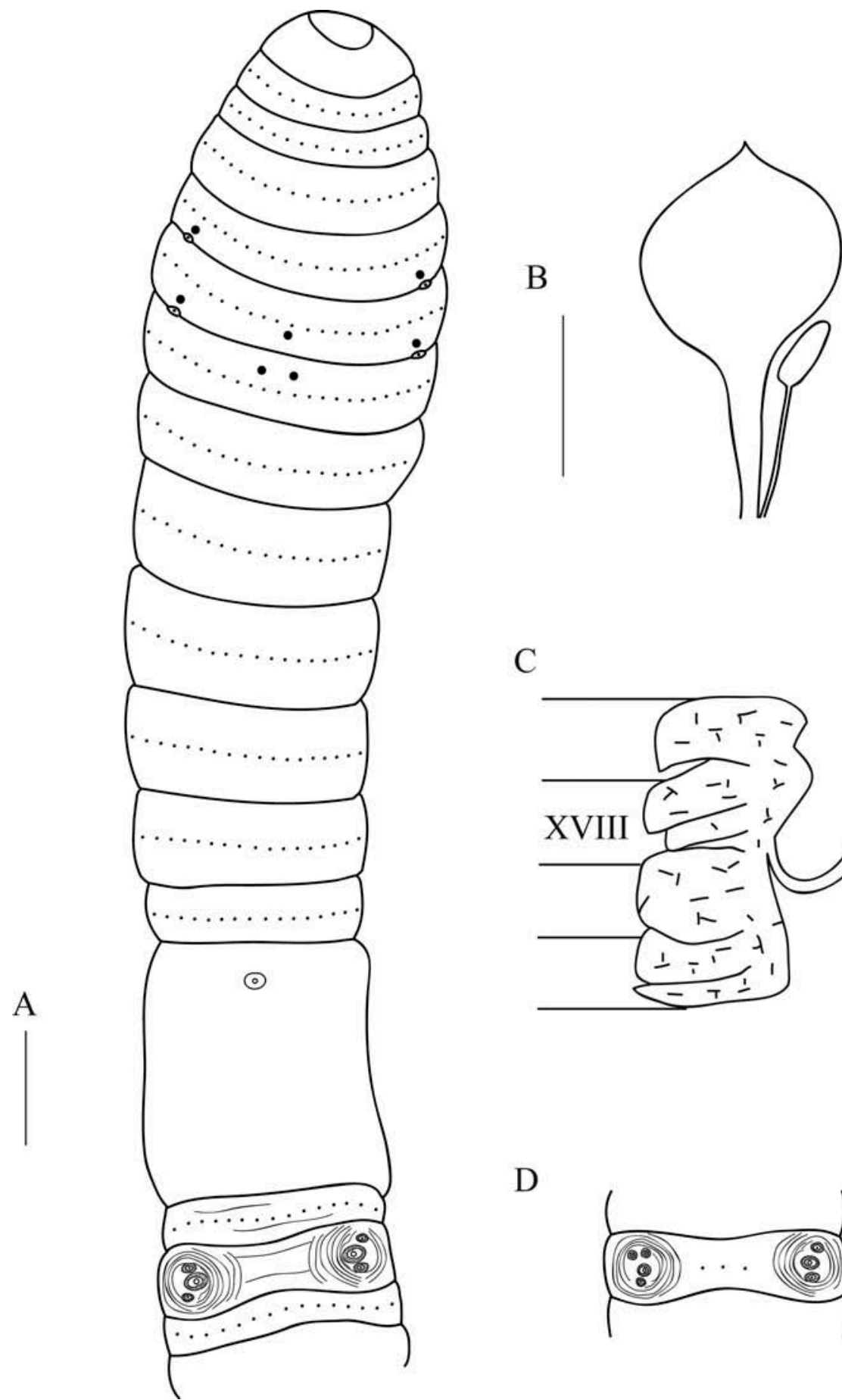

C

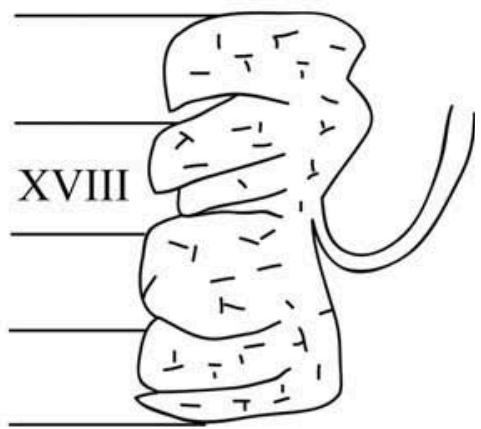

$\mathrm{D}$

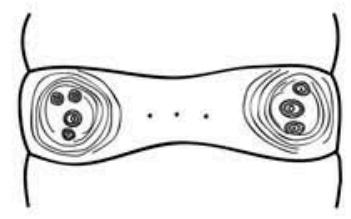

Figure 1. Amynthas instabilis Qiu and Jiang sp. nov. (A) Ventral view of holotype, scale bar $2 \mathrm{~mm}$; (B) spermathecae of holotype, scale bar $1 \mathrm{~mm}$; (C) prostate gland of holotype; (D) male pore region of one paratype. 
Zhang coll. 2 clitellates (C-GD201105-03): China, Guangdong Province, Xiangtoushan National Nature Reserve $\left(23^{\circ} 15^{\prime} 38^{\prime \prime} \mathrm{N}, 114^{\circ} 22^{\prime} 31^{\prime \prime} \mathrm{E}\right), 360$ m elevation, yellow soil under broadleaved evergreen forest, 23 October 2011, J.B. Jiang, J. Sun, J.X. Li and X.D. Lei coll.

Ten individuals were dissected and 13 were characterized externally only.

\section{Etymology}

The species is named after its characteristic of variable numbers of genital papillae in the male pore region.

\section{Diagnosis}

Dimensions $74-125 \mathrm{~mm}$ by $2.9-4.0 \mathrm{~mm}$ at clitellum, segments $82-145$. Setae numbering 30-54 at III, 42-64 at V, 52-62 at VIII, 40-52 at XX, 40-56 at XXV; 0-2 between male pores; $15-22$ between spermathecal pores. Spermathecal pores in 5/6-6/7, 0.40 body circumference ventrally apart. Male pores in XVIII, 0.33 body circumference ventrally apart, each on the top of a slightly raised, oval porophore in a pulvinate pad with three to six circular folds. Spermathecae two pairs in VI-VII, ampulla heartshaped, duct as long as ampulla. Diverticulum half as long as main pouch (duct and ampulla together), terminal one-third dilated into an ovoid chamber.

\section{External characters}

Taupe dorsal pigment, ventral pigment lighter present in the pre-clitellum segments of preserved specimens; tan pigment on dorsum, no pigment on ventrum of the postclitellum segments. Dimensions $74-125 \mathrm{~mm}$ by $2.9-4.0 \mathrm{~mm}$ at clitellum, segments $82-$ 145; the average values of examined individuals: dimensions $104 \mathrm{~mm}$ by $3.3 \mathrm{~mm}$ at clitellum, segments 128. Secondary annulations conspicuous in segments XVIIXVIII. Prostomium $1 / 2$ epilobous. First dorsal pore of all examined individuals in 11/12. Setae numbering 30-54 at III, 42-64 at V, 52-62 at VIII, 40-52 at XX, 40-56 at XXV; 0-2 between male pores; $15-22$ between spermathecal pores; setal formula: $\mathrm{AA}=1.0-1.3 \mathrm{AB}, \mathrm{ZZ}=1.0-2.0 \mathrm{ZY}$. Clitellum annular, pinkish or brown, in XIVXVI, setae invisible externally.

Spermathecal pores two pairs in 5/6-6/7, ventral, eye-like, sometime invisible, milky white porophore in centre, 0.40 circumference ventrally apart from each other. A tiny conical genital papilla before each pore. A similar postsetal genital papilla on midventral line of segment VI, and a pair of presetal genital papillae, $0.2 \mathrm{~mm}$ apart from each other, on the anterior border of segment VII.

Male pores one pair in XVIII, 0.33 circumference apart ventrally, each on the top of a slightly raised, oval porophore in a pulvinate pad with six circular folds, some paratypes with three circular folds. Two tiny oval genital papillae, present at anterior and posterior of porophore, each male pore is medial to these two tiny papillae (Figure 1A); some paratypes with three tiny oval genital papillae surrounding left male pore (Figure 1D). Genital papillae numbers in male pores region are variable among specimens.

Female pore single in XIV, rounded, milky white. 


\section{Internal characters}

Septa 4/5-6/7, 10/11 thick and muscular, 11/12 slightly thickened, 8/9-9/10 absent. Gizzard long bucket-shaped, in IX-X. Intestine enlarged distinctly from XVI. Intestinal caeca paired in XXVII, simple, smooth, with a slight indentation on terminal dorsal margin, extending anteriorly to XXIV. Oesophageal hearts in X-XIII.

Ovaries in XIII. Spermathecae two pairs in VI-VII, ampulla heart-shaped, about $2.3 \mathrm{~mm}$ long in holotype; ampulla duct slender to stout, as long as ampulla. Diverticulum as long as a half main pouch (duct and ampulla together), slender, terminal one-third dilated into an ovoid chamber (Figure 1B), milky white. No accessory glands observed.

Male sexual system holandric, testis sacs two pairs, in X-XI. Seminal vesicles two pairs, extending in XI-XIII, the posterior pair larger than the anterior one and extending to XIII. Prostate glands developed, inserting in XVIII and extending to $\mathrm{XVII}$ and $\mathrm{XX}$, coarsely lobate, prostatic duct U-shaped, slightly thicker at the distal part (Figure 1C). No accessory glands observed.

\section{Remarks}

In comparison to the other 30 species of the morrisi-group reported from China (19) and other Asian countries (11), Amynthas instabilis sp. nov. is closely related to Amynthas incongruus (Chen 1933) and Amynthas tripunctus (Chen 1946) from China, and to $A$. dilatatus sp. nov., A. infuscuatus sp. nov. and A. qiongzhongensis sp. nov. These six species share some similarities with similar male pores and genital markings, two pairs of intersegmental spermathecal pores in 5/6-6/7, and simple intestinal caeca. Table 3 illustrates in detail the differences among $A$. incongruus (Chen 1933), A. tripunctus (Chen 1946) and the four new species.

Amynthas instabilis sp. nov. differs from A. tripunctus (Chen 1946) by the presence of pigment on the dorsum, location of first dorsal pore, setae number in segment VIII, prostate glands and spermathecal characters. Amynthas instabilis sp. nov. has tan pigment on the dorsum, first dorsal pore in 11/12, setal number 52-62 at VIII, prostates glands less developed and diverticulum half as long as main pouch. In contrast, $A$. tripunctus has grey pigment on dorsum, first dorsal pore in 12/13, setae number fewer at VIII, more setae between male pores, well-developed prostate glands and a longer diverticulum.

Amynthas instabilis sp. nov. and A. incongruus (Chen 1933) can be separated on the basis of spermathecal characters. The ampulla of $A$. instabilis sp. nov. is heartshaped, a slender to stout duct is as long as ampulla. The ampulla of $A$. incongruus is ovoid or elongate spherical, duct either long and slender, or short stout, terminal three-quarters of diverticulum dilated into a twist chamber. In addition, $A$. incongruus has very small seminal vesicles and accessory glands, with prostate glands absent entirely and in a few cases well developed (Chen 1933).

Amynthas dilatatus Qiu and Jiang sp. nov.

(Figure 2)

\section{Material}

Holotype. 1 clitellate (C-HN012-03A): China, Hainan Island, Jianfengling National Nature Reserve (1843'26" N, 108 53'56" E), 900 m elevation, black 


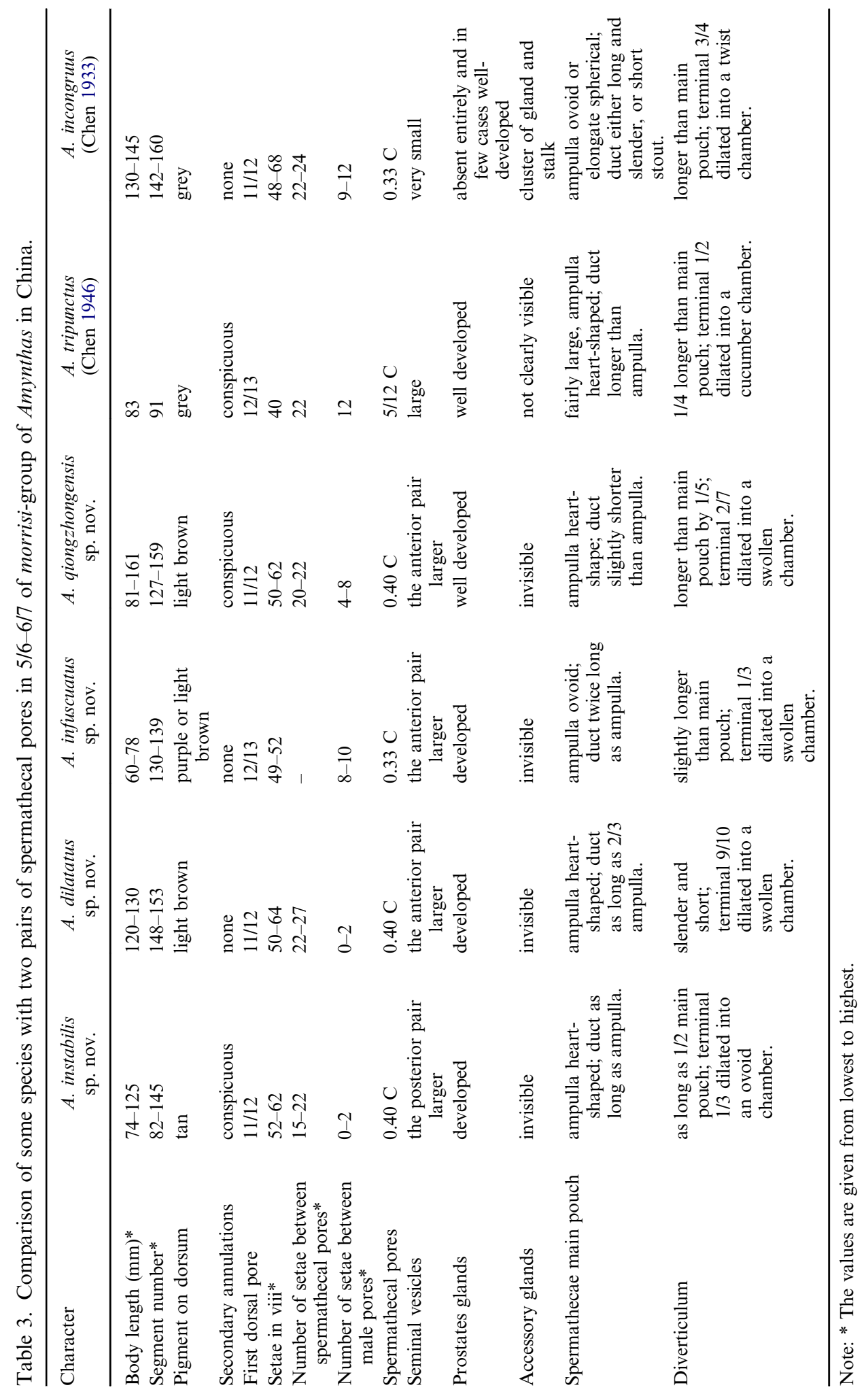




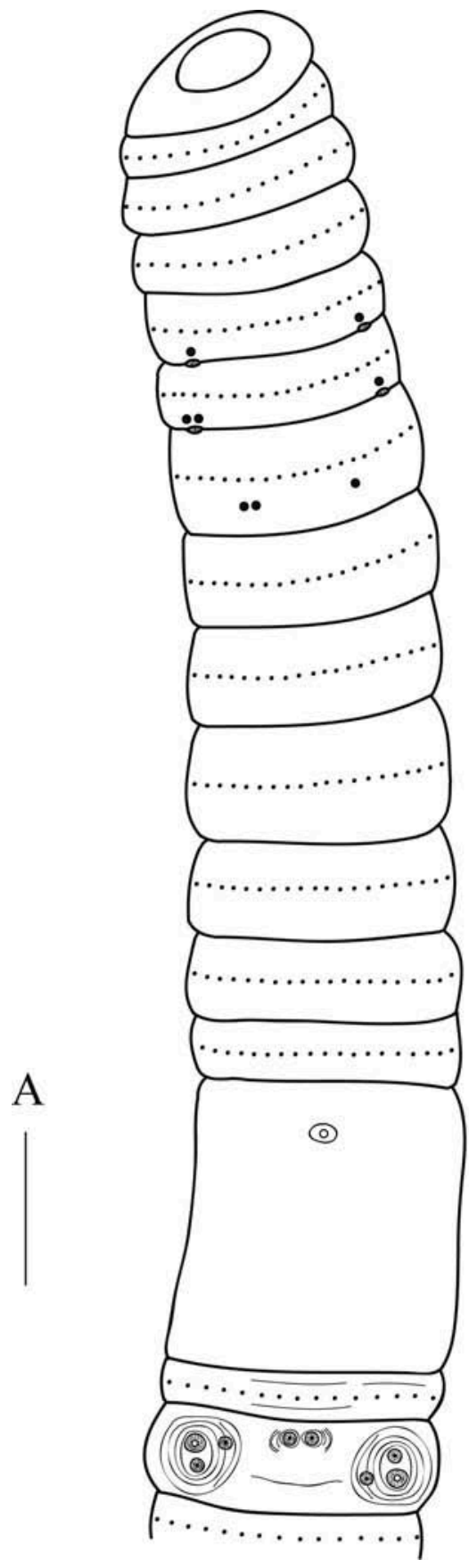

A

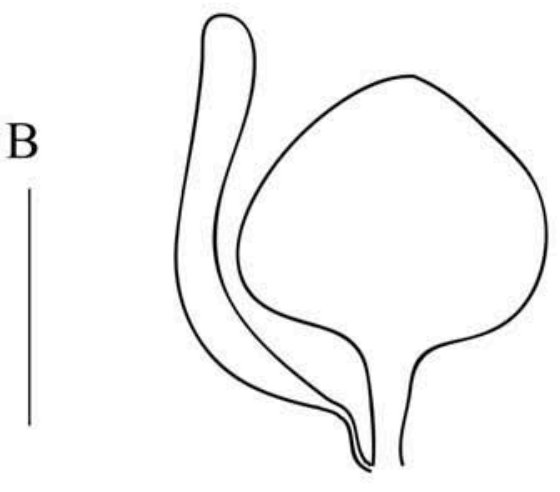

B

C

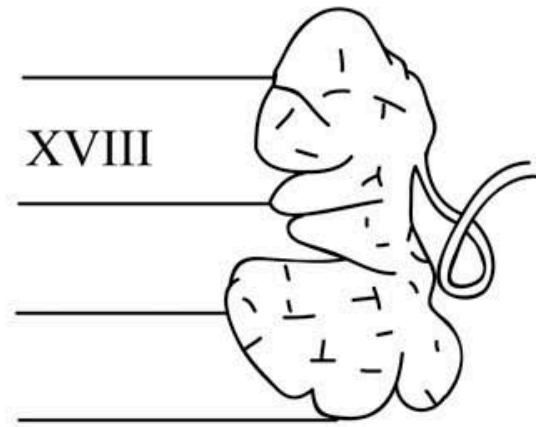

D

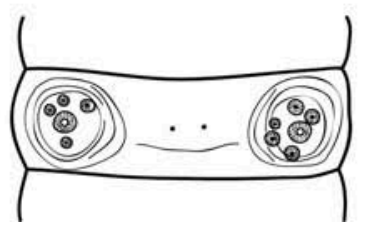

Figure 2. Amynthas dilatatus Qiu and Jiang sp. nov; (A) Ventral view of holotype, scale bar $2 \mathrm{~mm}$; (B) spermathecae of holotype, scale bar $1 \mathrm{~mm}$; (C) prostate gland of holotype; (D) male pore region of one paratype. 
sandy soil under shrubbery beside road, 4 July 2006, J.X. Li and W.X. Zhang coll.

Paratypes. 1 clitellate (C-HN012-03B) with the same data as for holotype. 7 clitellates (C-HN029-01): China, Hainan Island, Diaoluoshan National Nature Reserve $\left(18^{\circ} 43^{\prime} 31^{\prime \prime} \mathrm{N}, 109^{\circ} 52^{\prime} 01^{\prime \prime} \mathrm{E}\right), 920 \mathrm{~m}$ elevation, black sandy soil under meadow, 7 July 2006, J.P. Qiu, M.B. Bouché, J.X. Li and X.L. Zhang colls.

Both clitellates were externally characterized and dissected.

\section{Etymology}

The species is named after the characteristics of the seminal chamber.

\section{Diagnosis}

Dimensions $120-130 \mathrm{~mm}$ by $2.9-3.2 \mathrm{~mm}$ at clitellum, segments $148-153$. Setae numbering $46-50$ at III, 56-62 at V, 50-64 at VIII, 30-50 at XX, 41-50 at XXV; 0-2 between male pores; 22-27 between spermathecal pores. Spermathecal pores in $5 / 6-6 / 7,0.40$ body circumference ventrally apart. Male pores in XVIII, 0.33 body circumference ventrally apart, each on the top of a slightly raised, rounded porophore in a pulvinate pad with three or four circular folds. Spermathecae two pairs in VI-VII, ampulla heart-shaped, duct as long as two-thirds of ampulla. Diverticulum a little longer than the main pouch (duct and ampulla together), seminal chamber swollen.

\section{External characters}

Grey dorsal pigment present in the pre-clitellum segments of preserved specimens; light brown pigment on dorsum of the post-clitellum segments; no pigment on ventrum. Dimensions $120-130 \mathrm{~mm}$ by $2.9-3.2 \mathrm{~mm}$ at clitellum, segments 148-153; the average values of examined individuals: dimensions $125 \mathrm{~mm}$ by $3.0 \mathrm{~mm}$ at clitellum, segments 150 . Prostomium $1 / 2$ epilobous. First dorsal pore in 11/12. Setae numbering 46-50 at III, 56-62 at V, 50-64 at VIII, 30-50 at XX, 41-50 at XXV; 0-2 between male pores; 22-27 between spermathecal pores; setal formula: $\mathrm{AA}=1.0-1.2 \mathrm{AB}, \mathrm{ZZ}=1.0-1.8 \mathrm{ZY}$. Clitellum annular in XIV-XVI, light brown, swollen; setae invisible externally; gaps in dorsal could be identified clearly.

Spermathecal pores two pairs in 5/6-6/7, ventral, eye-like, 0.40 circumference ventrally apart from each other. One or two tiny conical genital papillae before each pore. Three similar presetal genital papillae irregularly present on posterior border of segments VII.

Male pores one pair in XVIII, 0.33 circumference ventrally apart from each other, each on the top of a slightly raised, rounded porophore in a pulvinate pad with three or four circular folds. each male pore is medial to these two tiny papillae (Figure 2A), some paratypes with four or five tiny oval genital papillae surrounding each male pore (Figure 2D). In holotype, a pair of tiny conical genital papillae present on anterior border of segments XVIII, $0.2 \mathrm{~mm}$ apart from each other.

Female pore single in XIV, ovoid, milky white. 
Internal characters

Septa 7/8-8/9 thick and muscular, 10/11-11/12 slightly thickened, 8/9-9/10 absent. Gizzard long bucket-shaped, in IX-X. Intestine enlarged distinctly from XV. Intestinal caeca paired in XXVII, simple, smooth, with two large indentation on dorsal and ventral margin, extending anteriorly to XXIV. Oesophageal hearts in X-XIII.

Ovaries in XIII. Spermathecae two pairs in VI-VII, ampulla heart-shaped, about $1.7 \mathrm{~mm}$ long in holotype; ampulla duct slender to stout, as long as two-thirds of ampulla. Diverticulum about $2.2 \mathrm{~mm}$ long, slender and short; seminal chamber swollen, about $2.0 \mathrm{~mm}$ (Figure 2B). No accessory glands observed.

Male sexual system holandric, testis sacs two pairs, in X-XI. Seminal vesicles two pairs, extending in X-XII, the pair in XI larger than the other. Prostate glands developed, inserting in XVIII and extending to $1 / 2 \mathrm{XVII}$ and XX, coarsely lobate composed of three major lobes. Prostatic duct U-shaped, conspicuously curved at the distal part (Figure 2C). No accessory glands observed.

\section{Remarks}

The present species is obviously different from the other species in Table 3 because of the long diverticulum with a swollen seminal chamber. In addition, A dilatatus sp. nov. differs from $A$. tripunctus (Chen 1946) in the features of body length, segment number, setae number between male pores, location of first dorsal pore and prostate glands.

Amynthas infuscuatus Jiang and Sun sp. nov.

(Figure 3)

\section{Material}

Holotype. 1 clitellate (C-HN020-02A): China, Hainan Island, Jianfengling National Nature Reserve (18 $\left.44^{\prime} 45^{\prime \prime} \mathrm{N}, 108^{\circ} 50^{\prime} 32^{\prime \prime} \mathrm{E}\right), 1020$ m elevation, cinnamon soil under tropical rainforest vegetation, 5 July 2006, J.P. Qiu, M.B. Bouché, J.X. Li and X.L. Zhang colls.

Paratypes. 2 clitellates (C-HN020-02B) with the same data as for holotype.

All three clitellates were externally characterized and dissected.

\section{Etymology}

The species is named after its dark pigment.

\section{Diagnosis}

Dimensions $60-78 \mathrm{~mm}$ by $1.4-1.6 \mathrm{~mm}$ at clitellum, segments $130-139$. Setae numbering $46-48$ at III, 56-60 at V, 49-52 at VIII, 42-46 at XX, 44-48 at XXV, 8-10 between male pores. Spermathecal pores two pairs in 5/6-6/7, 0.33 circumference ventrally apart from each other. Male pores in XVIII, 0.33 body circumference ventrally apart, each on the top of a slightly raised, oval porophore in a pulvinate 

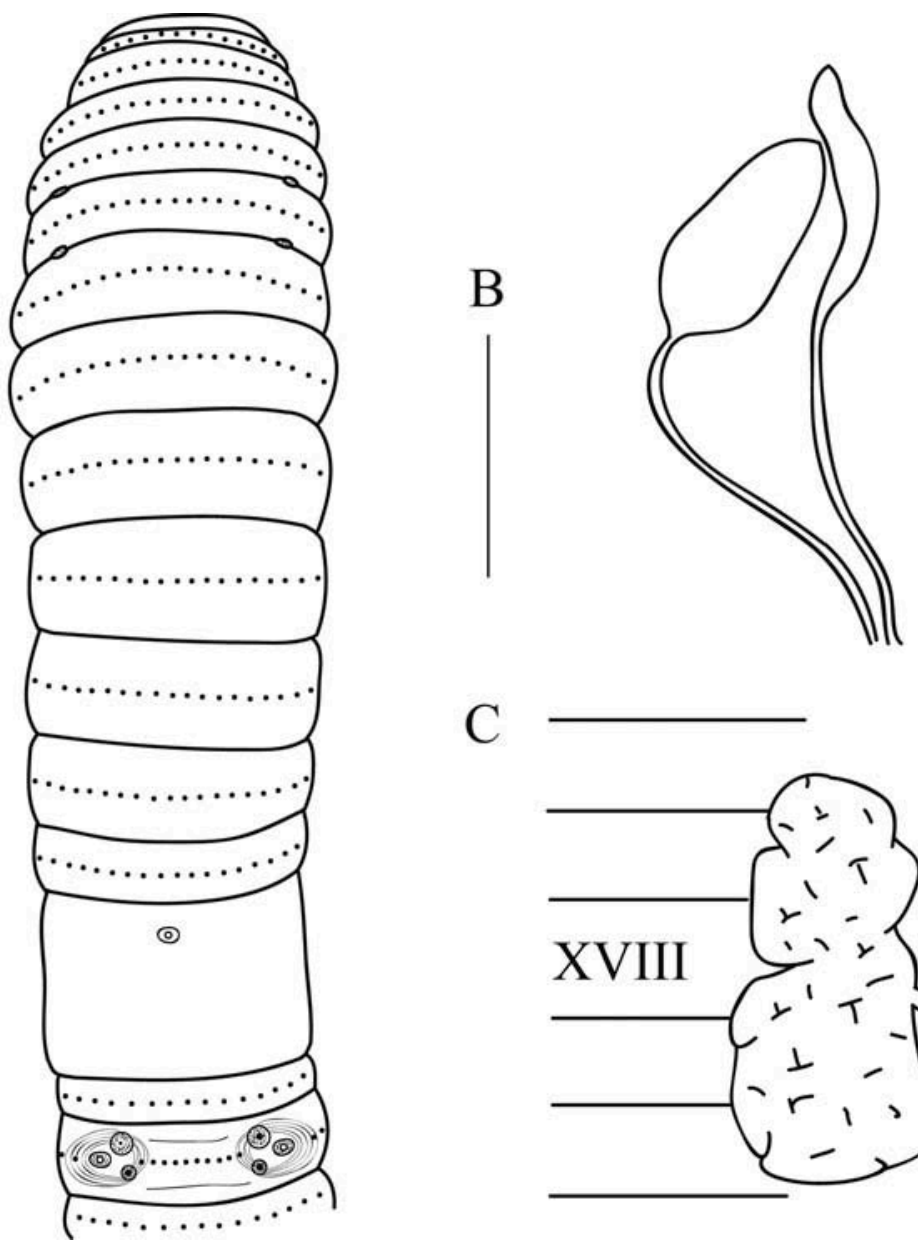

A
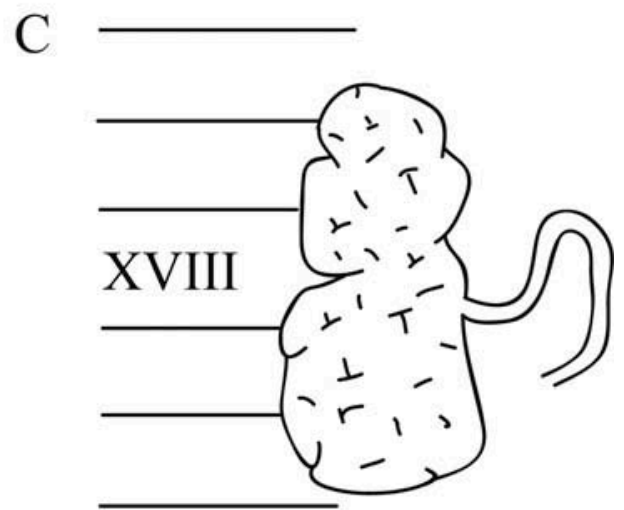

Figure 3. Amynthas infuscuatus Jiang and Sun sp. nov. (A) Ventral view of holotype, scale bar $2 \mathrm{~mm}$; (B) spermathecae of holotype, scale bar $1 \mathrm{~mm}$; (C) prostate gland of holotype.

pad with three to four circular folds. Spermathecae two pairs in VI-VII, ampulla ovoid, duct twice as long as ampulla. Diverticulum slightly longer than main pouch, slender, terminal one-third dilated into a swollen chamber.

\section{External characters}

Purple dorsal pigment before segment VIII of preserved specimens; light brown pigment on dorsum after segment VIII, no pigment on ventrum. Dimensions 60 $78 \mathrm{~mm}$ by $1.4-1.6 \mathrm{~mm}$ at clitellum, segments $130-139$; the average values of examined individuals: dimensions $68 \mathrm{~mm}$ by $1.5 \mathrm{~mm}$ at clitellum, segments 134 . Prostomium $1 / 2$ epilobous. First dorsal pore in 12/13. Setae numbering $46-48$ at III, 56-60 at V, 49-52 at VIII, 42-46 at XX, 44-48 at XXV; 8-10 between male pores; setal formula: $\mathrm{AA}=1.0-1.2 \mathrm{AB}, \mathrm{ZZ}=1.0-1.2 \mathrm{ZY}$. Clitellum annular, orange colour, in XIV-XVI, setae visible externally. 
Spermathecal pores two pairs in 5/6-6/7, ventral, eye-like, 0.33 circumference ventrally apart from each other.

Male pores one pair in XVIII, 0.33 circumference ventrally apart from each other, each on the top of a slightly raised, oval porophore in a pulvinate pad with three or four circular folds. Two rounded genital papillae, flat-topped, present at inner ridge of porophore, anteromedial one larger than the other (Figure 3A).

Female pore single in XIV, oval.

\section{Internal characters}

Septa 6/7-7/8 thick and muscular, 10/11-12/13 slightly thickened, 8/9-9/10 absent. Gizzard long bucket-shaped, in IX-X. Intestine enlarged distinctly from XVI. Intestinal caeca paired in XXVII, simple, smooth, brown, extending anteriorly about to XXIV. Oesophageal hearts in X-XIII.

Ovaries in XIII. Spermathecae two pairs in VI-VII, ampulla ovoid, about $2.5 \mathrm{~mm}$ long in holotype; duct slender, twice as long as ampulla. Diverticulum slightly longer than main pouch, slender, terminal one-third dilated into a swollen chamber (Figure 3B), milky white. No accessory glands observed.

Male sexual system holandric, testis sacs two pairs, in X-XI. Seminal vesicles two pairs, extending in $\mathrm{X}-\mathrm{XII}$, the anterior pair larger. Testis sacs and seminal vesicles separated from each other on ventrum. Prostate glands developed, inserting in XVIII and extending to XVI and half of XX, coarsely lobate composed of two major lobes. Prostatic duct U-shaped, slender (Figure 3C). No accessory glands observed.

\section{Remarks}

Both $A$. infuscuatus sp. nov. and A. tripunctus have similar body length, setae, location of first dorsal pore, seminal vesicles and diverticulum length. However, A. infuscuatus sp. nov. is distinguished from A. tripunctus by its segment number, ampulla shape and seminal chamber length.

In comparison to $A$. incongruus, spermathecae of $A$. infuscuatus sp. nov. is quite different. Ampulla is heart-shaped, duct slightly shorter than ampulla, and terminal two-sevenths of diverticulum dilated into a swollen seminal chamber. Moreover, body length and first dorsal pore are also distinctive characters.

Although both have terminal third dilated into seminal chamber, A. infuscuatus sp. nov. and $A$. instabilis have no similar spermathecae. The ampulla of $A$. infuscuatus is ovoid, smaller than the heart-shaped ampulla of $A$. instabilis, but its ampulla duct and diverticulum are longer.

Amynthas qiongzhongensis Jiang and Zhao sp. nov. (Figure 4)

\section{Material}

Holotype. 1 clitellate (C-HN022-02A): China, Hainan Island, Diaoluoshan National Nature Reserve (18 43'30" N, 109 52'07" E), 920 m elevation, brown sandy soil under shrubbery, 6 July 2006, J.P. Qiu, M.B. Bouché, J.X. Li and X.L. Zhang colls. 
14 J. Jiang et al.

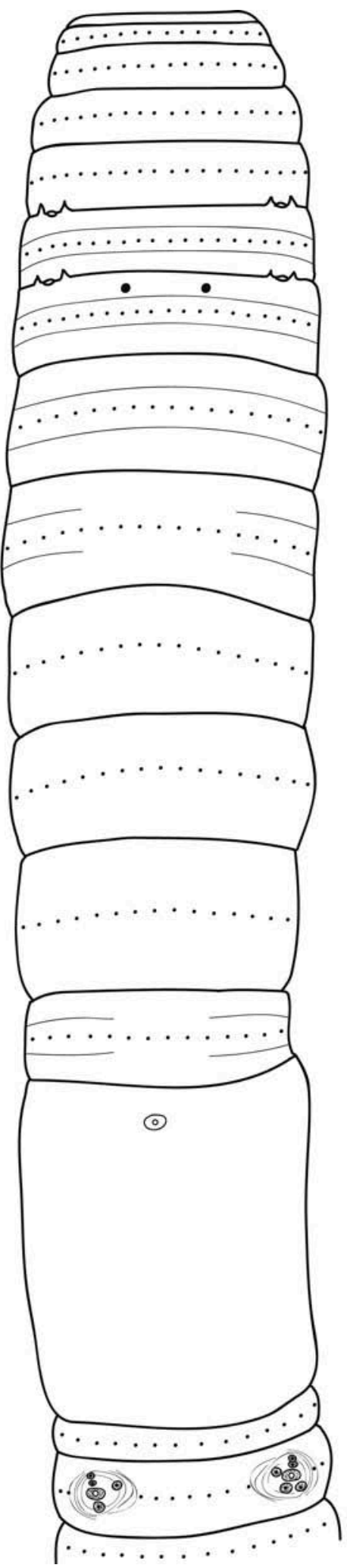

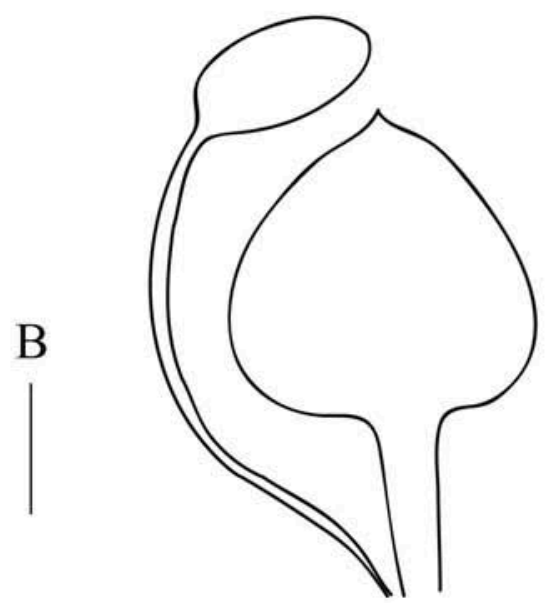

C

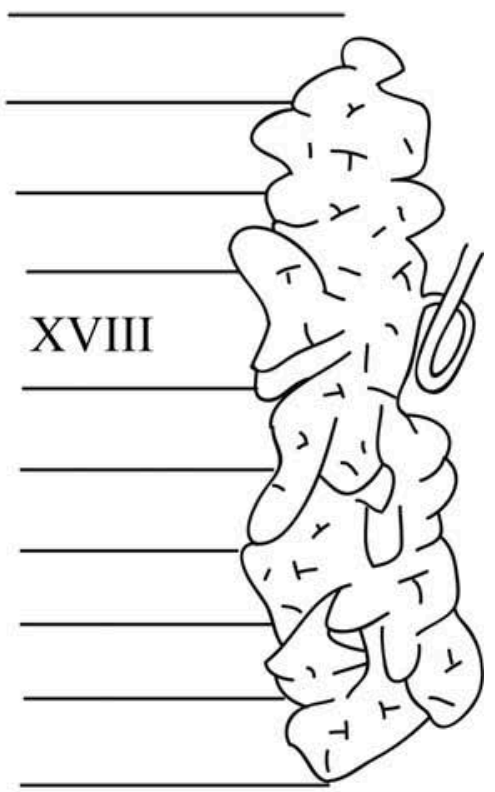


Paratypes. 9 clitellates (C-HN022-02B) with the same data as for holotype. 2 clitellates (C-HN201008-01), 2 clitellates (C-HN201008-02): China, Hainan Island, Diaoluoshan National Nature Reserve (1843'35" N, 109 52'02" E), 934 m elevation, black soil under palm trees, 24 July 2010, J.B. Jiang and Y.Z. Guo coll. 1 clitellate (C-HN201012-02): China, Hainan Island, Diaoluoshan National Nature Reserve $\left(18^{\circ} 43^{\prime} 15^{\prime \prime} \mathrm{N}, 109^{\circ} 52^{\prime} 18^{\prime \prime} \mathrm{E}\right), 933$ m elevation, black soil under scrubs, 24 July 2010, J.B. Jiang and Y.Z. Guo coll. 1 clitellate (C-HN201114-02): China, Hainan Island, Limushan Nature Reserve, Quling Mountain (19¹3'40" N, 10944'15" E), 739 m elevation, 27 May 2011, J.P. Qiu, J.B. Jiang, Q. Zhao, D. Cluzeau and W.K. Zhang coll.

Six individuals were dissected and 10 individuals were characterized externally only.

\section{Etymology}

The species is named after its type locality (Li-Miao Autonomous County of Qiongzhong, Hainan).

\section{Diagnosis}

Dimensions $81-161 \mathrm{~mm}$ by $3.4-4.6 \mathrm{~mm}$ at clitellum, segments $127-159$. Setae numbering $42-46$ at III, 48-64 at V, 50-62 at VIII, 40-54 at XX, 40-80 at XXV; 4-8 between male pores; $21-26$ between spermathecal pores. Spermathecal pores in 5/6-6/7, 0.40 body circumference ventrally apart. Male pores in XVIII, 0.33 body circumference ventrally apart, each on the top of a slightly raised, oval porophore in a pulvinate pad with three to five circular folds. Spermathecae two pairs in VIVII, ampulla heart-shaped, duct slightly shorter than ampulla. Diverticulum is longer than the main pouch by one-fifth, terminal two-sevenths dilated into a swollen chamber.

\section{External characters}

Grey dorsal pigment present in the pre-clitellum segments of preserved specimens; light brown pigment on dorsum of the post-clitellum segments; no pigment on ventrum. Dimensions $81-161 \mathrm{~mm}$ by $3.4-4.6 \mathrm{~mm}$ at clitellum, segments $127-159$; the average values of examined individuals: dimensions $125 \mathrm{~mm}$ by $3.9 \mathrm{~mm}$ at clitellum, segments 147. Secondary annulations conspicuous in pre-clitellum segments. Prostomium combined prolobous and $1 / 2$ epilobous. First dorsal pore in $11 /$ 12. Setae numbering 42-46 at III, 48-64 at V, 50-62 at VIII, 40-54 at XX, 40-80 at $\mathrm{XXV}$; 4-8 between male pores; $21-26$ between spermathecal pores; setal formula: $\mathrm{AA}=1.0-1.5 \mathrm{AB}, \mathrm{ZZ}=1.0-2.0 \mathrm{ZY}$. Clitellum annular, purple or light brown colour, in XIV-XVI, setae invisible externally.

Figure 4. Amynthas qiongzhongensis Jiang and Zhao sp. nov. (A) Ventral view of holotype, scale bar $2 \mathrm{~mm}$; (B) spermathecae of holotype, scale bar $1 \mathrm{~mm}$; (C) prostate gland of holotype. 
Spermathecal pores two pairs in 5/6-6/7, ventral, 0.40 circumference apart from each other. A couple of antrorse genital papillae surround each spermathecal pore. A pair of tiny, collapse-topped, presetal genital papillae present on anterior border of segments VII.

Male pores one pair in XVIII, 0.33 circumference ventrally apart from each other, each on the top of a slightly raised, oval porophore in a pulvinate pad with three to five circular folds. Four or five genital papillae tiny, collapse-topped, surrounding each male pore, present on the porophore (Figure 4A).

Female pore single in XIV, rounded, milky white.

\section{Internal characters}

Septa 5/6-7/8 thick and muscular, 10/11-12/13 slightly thickened, 8/9-9/10 absent. Gizzard short bucket-shaped, in 1/2VIII-IX. Intestine enlarged distinctly from XVI. Intestinal caeca paired in XXVII, simple, smooth, extending anteriorly to XXIV. Oesophageal hearts in X-XIII.

Ovaries in XIII. Spermathecae two pairs in VI-VII, ampulla heart-shaped, about $2.8 \mathrm{~mm}$ long in holotype; stout duct slightly shorter than ampulla. Diverticulum is longer than the main pouch by one-fifth, slender, terminal twosevenths dilated into a swollen chamber (Figure 4B), milky white. No accessory glands observed.

Male sexual system holandric, testis sacs two pairs, in X-XI. Seminal vesicles two pairs, extending in XI-XII, the anterior pair well developed. Prostate glands well developed, inserting in XVIII and extending to $1 / 2 \mathrm{XV}$ and XXIII, coarsely lobate composed of several major lobes. Prostatic duct U-shaped, slender, conspicuously curved at the distal part (Figure 4C). No accessory glands observed.

\section{Remarks}

The new species is similar to A. tripunctus in having similar body length, body width, prostate glands and ampulla shape, but it has a shorter seminal chamber, while the terminal half of the diverticulum of $A$. tripunctus is dilated into a cucumber-shaped chamber.

Amynthas qiongzhongensis sp. nov. differs greatly from $A$. incongruus by the characteristics of the accessory glands and spermathecae.

Amynthas qiongzhongensis sp. nov. differs from $A$. instabilis sp. nov. in the features of spermathecae, prostate glands and other characters.

\section{Acknowledgements}

This study was supported by the National Science Foundation of China grant No. 31172360 and No. 31272265. We are grateful to Professor Marcel Bouché (Laboratoire de Zooecologie du sol, Montpellier, France), Professor Daniel Cluzeau (Université de Rennes, France), Dr Jianxiong Li (Guangdong Entomological Institute), Professor Xingliang $\mathrm{Wu}$ and Mrs Jingxia Zhong (Hainan University), Dr Weixin Zhang (South China Botanical Garden), Yongzhi Guo, Xiande Lei and Wenkun Zhang for their assistance with the field work. We thank the reviewers of the manuscript for many useful suggestions and corrections. 


\section{References}

Beddard FE. 1892. On some species of the genus Perichoeta (sensu strocto). Proc Zool Soc. $153-172$.

Bely AE, Wray GA. 2004. Molecular phylogeny of naidid worms (Annelida: Clitellata) based on cytochrome oxidase I. Mol Phylogenet Evol. 30:50-63.

Chang CH, James S. 2011. A critique of earthworm molecular phylogenetics. Pedobiologia. 54: SS3-SS9.

Chen Y. 1933. A preliminary survey of the earthworms of the lower Yangtze Valley. Contrib Biol Lab Sci Soc China (Zool). 9:177-295.

Chen Y. 1938. Oligochaeta from Hainan, Kwangtung. Sci Soc China. Contrib Biol Lab Sci Soc China (Zool). 12:375-427.

Chen Y. 1946. On the terrestrial Oligochaeta from Szechuan III. Bor Res Soc J West China Border Res Soc. 16:83-141.

Chen Y, Hsu C. 1977. On some new earthwoms from China II. Curr Zool. 23:175-181.

Chen Y, Hsu C, Yang T, Fong H. 1975. On some new earthwoms from China. Curr Zool. 21:89-99.

Folmer RHA, Nilges M, Folkers PJM, Konings, RNH, Hilbers, CW. 1994. A model of the complex between single-stranded DNA and the single-stranded DNA binding protein encoded by gene V of filamentous bacteriophage M13. J Mol Biol. 240:341-357.

Hong Y. 2007. Some new earthworms of the genus Amynthas (Oligochaeta: Megascolecidae) with male discs from Bogildo Island, Korea. Revue Suisse De Zoologie. 114:721-728.

Hong Y, James SW. 2001. New species of Korean Amynthas Kinberg, 1867 (Oligochaeta, Megascolecidae) with two pairs of spermathecae. Revue Suisse De Zoologie. 108:65-93.

Hong Y, James SW. 2009. Some new Korean Megascolecoid earthworms (Oligochaeta). J Nat Hist. 43:1229-1256.

Hong Y, Kim TH. 2002. Four new earthworms of the genus Amynthas (Oligochaeta: Megascolecidae) from Korea. Korean J Biol Sci. 6:195-199.

Huang J, Xu Q, Sun ZJ, Tang GL, Su ZY. 2007. Identifying earthworms through DNA barcodes. Pedobiologia. 51:301-309.

Novo M, Almodóvar A, Fernández R, Trigo, D, Díaz Cosín, DJ. 2010. Cryptic speciation of hormogastrid earthworms revealed by mitochondrial and nuclear data. Mol Phylogenet Evol. 56:507-512.

Sims RW, Easton EG. 1972. A numerical revision of the earthworm genus Pheretima auct. (Megascolecidae: Oligochaeta) with the recognition of new genera and an appendix on the earthworms collected by the Royal Society North Borneo Expedition. Biol J Linn Soc. 4:169-268.

Sun J, Zhao Q, Qiu JP. 2009. Four new species of earthworms belonging to the genus Amynthas (Oligochaeta: Megascolecidae) from Diaoluo Mountain, Hainan Island, China. Revue Suisse De Zoologie. 116:289-301.

Zhao Q, Sun J, Jiang JB, Qiu JP. 2013. Four new species of genus Amynthas (Oligochaeta, Megascolecidae) from Hainan Island, China. J Nat Hist. 47:2175-2192. 